\title{
PREENCHIMENTO DA CADERNETA DE SAÚDE DA PESSOA IDOSA: RELATO DE EXPERIÊNCIA
}

COMPLETION OF THE ELDERLY PERSON'S HEALTH RECORD: AN EXPERIENCE REPORT

LLENADO DE LA LIBRETA DE SALUd DEL ANCIANO: UN RELATO DE EXPERIENCIA

Alessandra Schmidt ${ }^{1}$

Cenir Gonçalves Tier ${ }^{2}$

Maria Eduarda Deitos Vasquez ${ }^{3}$

Vanessa Alvez Mora da Silva 4

Caroline Bittencourt ${ }^{5}$

Bethânia Mesquita Cabeda Maciel 6

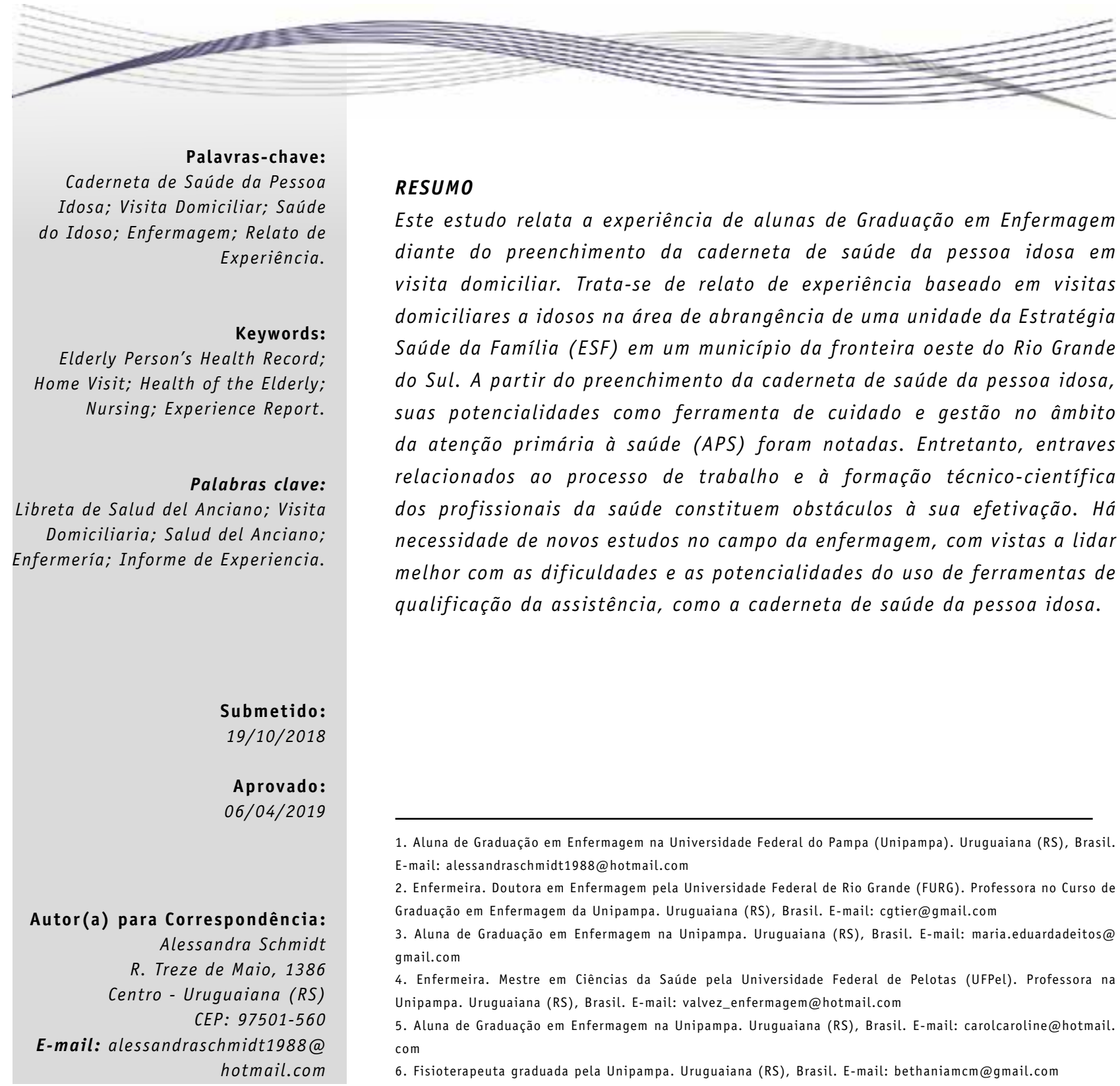

98 - SANARE, Sobral - V.18 n.01,p.98-106, Jan./Jun. - 2019 


\section{ABSTRACT}

This study reports the experience of female undergraduate Nursing students when completing the elderly person's health record during a home visit. This is an experience report based on home visits to the elderly within the area covered by a Family Health Strategy (FHS) center in a municipality located on the western border of the State of Rio Grande do Sul, Brazil. By completing the elderly person's health record, its potentialities as a tool for health care and management in the scope of primary health care (PHC) were noticed. However, hindrances related to the work process and to the technical-scientific qualification of health professionals constitute obstacles to its deployment. There is a need for further studies in the field of nursing, in order to better deal with the difficulties and potentialities of using health care qualification tools, such as the elderly person's health record.

\section{RESUMEN}

Este estudio relata la experiencia de alumnas de Graduación en Enfermería ante el llenado de la libreta de salud del anciano durante visita domiciliaria. Este es un informe de experiencia basado en visitas domiciliarias a ancianos en el área cubierta por un centro de la Estrategia Salud de la Familia (ESF) en un municipio ubicado en la frontera oeste del Estado de Rio Grande do Sul, Brasil. Al llenar la libreta de salud del anciano, sus potencialidades como herramienta para el cuidado y la gestión de la salud en el ámbito de la atención primaria de salud (APS) fueron percibidas. Sin embargo, trabas relacionadas con el proceso de trabajo y la calificación técnico-científica de profesionales de la salud constituyen obstáculos a su efectividad. Hay necesidad de nuevos estudios en el campo de la enfermería, con miras a lidiar mejor con las dificultades y las potencialidades del uso de herramientas de calificación de la atención, como la libreta de salud del anciano.

\section{INTRODUÇÃ O}

Segundo a Organização Mundial da Saúde (OMS), os idosos são definidos como pessoas com idade cronológica de 60 anos ou mais (em países em desenvolvimento) ou 65 anos ou mais (em países desenvolvidos) - mesmo que a idade cronológica não represente um marcador preciso das alterações decorrentes do envelhecimento, pois as mudanças relativas a estado de saúde, participação e nível de independência são perpassadas pelas singularidades dos indivíduos ${ }^{1}$.

As estimativas de recente estudo do Instituto Brasileiro de Geografia e Estatística (IBGE) refletem uma acelerada intensificação da proporção de idosos: demonstrou-se que, entre 2005 e 2015, esse grupo etário passou de $9,8 \%$ para $14,3 \%$ da população, em crescimento paralelo ao decréscimo do grupo etário de 0 a 14 anos - que passou de $26,5 \%$ para $21,0 \%{ }^{2}$.

Tais mudanças evidenciadas na composição demográfica já começam a provocar consequências sociais, culturais e epidemiológicas, sobretudo no que diz respeito ao perfil de morbimortalidade, a partir do aumento da incidência de doenças crônicodegenerativas. Esse fato suscita um olhar atento dos gestores e dos serviços de saúde, a fim de prevenir agravos e promover, proteger e recuperar a saúde da população idosa $a^{3,4}$.

A mudança do perfil de saúde da população idosa trouxe a necessidade de alterações nas formas de cuidado dessa população; uma delas foi o retorno ao modelo de cuidados domiciliares. Entre as diversas ações criadas para dar corpo a esse novo modelo, em consonância com a Política Nacional de Saúde da Pessoa Idosa (PNSPI), de 2006, destaca-se a prática sistemática de visitas domiciliárias, realizadas pelas equipes da Estratégia Saúde da Família (ESF) ${ }^{5}$.

Também em 2006, com o propósito de pactuar novos compromissos e responsabilidades entre as três esferas de gestão pública (federal, estadual e municipal), o Ministério da Saúde (MS) aprovou o "Pacto pela Saúde" do Sistema Único de Saúde (SUS), composto por: "Pacto em Defesa do SUS"; "Pacto de Gestão" e "Pacto pela Vida"6.

0 Pacto pela Vida indica a saúde do idoso como um de seus elementos prioritários, abrangendo diretrizes operacionais e ações estratégicas, coletivas e individuais. No conjunto de iniciativas por ele estabelecido, alinhado à implantação da PNSPI, encontra-se a implementação da caderneta de saúde da pessoa idosa ${ }^{7}$.

Esse instrumento configura-se como uma ferramenta para a qualificação da atenção e do manejo das demandas de saúde do idoso, uma vez que promove o levantamento periódico de determinadas condições e outros aspectos que possam interferir no bem-estar desse indivíduo, a fim de possibilitar que as ações necessárias sejam adotadas precocemente?.

A relevância deste estudo se justifica em tal contexto, tendo em vista que o profissional da ESF 
deve realizar a consulta de enfermagem e preencher a caderneta de saúde da pessoa idosa, contribuindo para a prevenção de agravos e a promoção e proteção da saúde, bem como para a recuperação e reabilitação do indivíduo, da família e da comunidade ${ }^{8}$.

Este artigo relata a experiência de alunas de Graduação em Enfermagem diante do preenchimento da caderneta de saúde da pessoa idosa em visita domiciliar.

\section{METODOLOGIA}

Trata-se de relato de experiência construído a partir das vivências de acadêmicas de Enfermagem de uma universidade federal localizada na fronteira oeste do Rio Grande do Sul, durante as aulas práticas da disciplina “Enfermagem no Cuidado à Saúde do Idoso", ambientadas em um serviço de atenção primária à saúde (APS).

Esse componente curricular tem por objetivos:

A. Capacitar os alunos para a assistência qualificada ao idoso nos diferentes níveis de atenção à saúde;

B. Fomentar reflexões sobre a inserção do idoso na sociedade contemporânea;

C. Conhecer as políticas públicas nacionais e os serviços de atenção ao idoso; e

D. Identificar o idoso ativo ou fragilizado/ doente, estimulando a promoção à sua saúde e a manutenção de sua funcionalidade e autonomia.

Assim, este relato se concentra em visitas domiciliares a idosos adstritos ao território de uma unidade da ESF, localizada na zona urbana do município, que conta com 2 equipes compostas por: 2 enfermeiros; 2 médicos generalistas; 3 técnicos de enfermagem; 1 nutricionista; 1 educador físico; e 8 agentes comunitários de saúde (ACS). Esse serviço de saúde abrange 13 microáreas e atende, segundo o Sistema de Informações da Atenção Básica (SIAB), uma população de 8.800 pessoas ( $35 \%$ delas com 60 anos ou mais).

Nesse serviço, o atendimento à comunidade ocorre em 2 turnos (das 7:30 às 11:30 e das 13:30 às 17:30) e estrutura-se mediante acolhimento à livre demanda, segundo os critérios da classificação de risco.

Para a operacionalização das atividades práticas, desenvolvidas em abril e maio de 2017, as acadêmicas se dividiram em duplas para as visitas domiciliares aos idosos, com o intuito de realizar o preenchimento da caderneta de saúde da pessoa

\section{...favorece ações de rastreamento e identificação da predisposição e do grau de fragilidade do idoso...}

idosa - em processo de implantação no município, mediante acompanhamento dos ACS e da professora responsável pela disciplina.

Ao longo desse período foram realizadas duas visitas a domicílio, de usuários adstritos no território. Na residência foram ofertadas ações de educação em saúde, imunização, aferição dos sinais vitais, além de distribuição, preenchimento e orientações a respeito da caderneta.

\section{RESULTADOS E DISCUSSÃO}

Optou-se por relatar as experiências das acadêmicas quanto ao preenchimento da caderneta de saúde da pessoa idosa agrupando aprendizados, resultados e limites identificados no desenvolvimento dessa atividade.

Discute-se o uso desse instrumento na APS destacando seu potencial enquanto ferramenta de cuidado e gestão, bem como os entraves observados no processo de sua implementação em uma unidade de ESF.

Apresentamos a seguir duas categorias temáticas oriundas das experiências das acadêmicas.

\section{Caderneta de saúde da pessoa idosa: ferramenta de cuidado e gestão}

No que concerne à caderneta de saúde da pessoa idosa, uma das potencialidades observadas pelas acadêmicas ao longo das atividades práticas na comunidade diz respeito à sua estruturação sistematizada, que favorece ações de rastreamento e identificação da predisposição e do grau de fragilidade do idoso a partir de seu preenchimento pela equipe da ESF, por concentrar informações de modo organizado e dinâmico.

Para fins operacionais, a PNSPI estratificou a população idosa em dois grupos compostos, respectivamente, por idosos independentes, ativos 
no meio familiar e social, e por aqueles considerados frágeis ou em processo de fragilização?.

0 segundo grupo vivencia certa dificuldade para realizar suas atividades da vida diária (AVD), o que pode resultar na perda de autonomia e na necessidade de cuidados e tratamentos contínuos ${ }^{10}$. As alterações funcionais próprias do envelhecimento, associadas à maior prevalência das doenças crônicodegenerativas, coadunam para a deterioração da independência, o que corrobora a diminuição do bem-estar e da qualidade de vida ${ }^{11}$.

Nesse sentido, a caderneta, como ferramenta do cuidado, surge com o propósito de identificar o grupo fragilizado no território, além de proporcionar - acompanhamento periódico de determinadas condições de saúde do idoso e outros aspectos que possam interferir em seu bem-estar, de modo a possibilitar o desenvolvimento de ações prioritárias de saúde voltadas à recuperação, promoção, atenção e prevenção de agravos entre esses indivíduos ${ }^{9}$.

0 primeiro contato das acadêmicas com a caderneta ocorreu ainda em sala de aula, durante uma dinâmica proposta pelas professoras. Nesse momento, a turma se organizou em duplas, que deveriam representar uma interação entre o enfermeiro e o idoso no contexto da visita domiciliar, para a simulação do preenchimento dos itens que compõem a caderneta.

No que diz respeito aos segmentos abarcados em sua estrutura, pode-se destacar, inicialmente, um espaço dedicado à obtenção de informações relativas à identificação do idoso que devem ser colhidas junto ao indivíduo, seus familiares e cuidadores, para garantir a veracidade dos dados. Esse fato dá início à execução do cuidado personalizado, uma vez que permite que as vulnerabilidades, sejam elas sociais, econômicas ou familiares, sejam explicitadas e (re)conhecidas pelos profissionais que realizam o acompanhamento periódico $0^{9,12,13}$.

$\mathrm{Na}$ sequência, o instrumento apresenta seções destinadas à coleta de informações individuais e familiares, objetivando o (re)conhecimento das redes de apoio, as interações e os relacionamentos. No espaço subsequente devem ser registradas informações estratégicas, que se relacionam às condições de saúde do idoso, especialmente, associadas a maior risco de desfechos adversos, considerando as intervenções preventivas de declínio funcional, hospitalização ou óbito ${ }^{9,12,13}$.

Ao longo do preenchimento da caderneta surgem questões gerais para a avaliação da saúde do idoso, que incluem o uso de medicamentos,

\section{...o instrumento \\ apresenta seções destinadas à coleta de informações individuais $e$ familiares...}

fitoterápicos, suplementos e vitaminas, em caráter contínuo ou ocasional; diagnósticos e internações prévias; procedimentos cirúrgicos realizados; reações adversas ou alergia a fármacos; e dados antropométricos ${ }^{9}$.

0 caderno de envelhecimento e saúde da pessoa idosa de 2007, em paralelo à caderneta, igualmente ratifica a importância do registro das medicações, considerando a necessidade de prevenção da ocorrência de iatrogenias medicamentosas, relacionadas à polifarmácia, ou uso concomitante de cinco medicamentos ou mais, comum diante da terapêutica de doenças crônicas ${ }^{12}$. Nesse sentido, um estudo descritivo realizado no Estado do Ceará junto a 134 idosos adstritos em uma unidade básica de saúde (UBS) constatou prevalência de polifarmacoterapia em $82,1 \%$ dos casos, referindo como condições crônicas mais comuns a hipertensão arterial $(59,1 \%)$ e o diabetes mellitus $(25,2 \%)^{14}$.

Por sua vez, uma pesquisa piauiense revelou que $62,5 \%$ dos participantes, com idades entre 60 e 80 anos utilizam medicamentos sem prescrição e que $42,5 \%$ responderam positivamente quanto à dificuldade para o uso de medicações nos horários e nas doses corretas. Logo, conhecer as práticas de administração dos fármacos e suas características nos idosos é essencial para que os profissionais da saúde possam prestar orientações efetivas a essa população ${ }^{15}$.

Outros pontos em evidência nesta seção se relacionam à aplicação do protocolo de identificação do idoso vulnerável (VES-13), no qual se utiliza um questionário que busca identificar o idoso vulnerável residente na comunidade, com base em idade, autopercepção da saúde, presença de limitações físicas e incapacidades. Há informações complementares, relativas ao humor e ao esquecimento autorreferidos; à avaliação ambiental, associada a elementos externos capazes de precipitar quedas; às quedas, ao histórico de quedas 
e aos desfechos associados; à identificação de dor crônica; e aos hábitos de vida ${ }^{12}$.

Um estudo realizado em Pernambuco, em maio de 2016, baseado no protocolo do instrumento VES-13, revelou que sua aplicação se mostrou confiável em termos da solidez e consistência de suas medidas, apresentando uma estrutura simples e de fácil execução que auxilia na identificação de pessoas idosas vulneráveis que necessitam de acompanhamento prioritário e constante dos serviços de saúde ${ }^{16}$. Outro estudo aponta a importância da autoavaliação como ferramenta de investigação ampliada do estado de saúde do idoso, auxiliando a compreensão do processo saúde-doença sob a ótica do próprio indivíduo ${ }^{17}$.

No que tange à avaliação ambiental, percebe-se que os fatores de risco ambientais ou extrínsecos, que aumentam a propensão dos idosos a quedas e acidentes domésticos, como iluminação, tapetes soltos, degraus altos ou estreitos, demandam verificação contínua por parte dos profissionais das equipes de saúde, com o objetivo de prevenir quedas e orientar quanto às modificações necessárias para obter maior segurança.

Nesse sentido, uma pesquisa realizada com 251 idosos identificou que $54 \%$ das quedas apresentadas por esse grupo tiveram como principal causa o ambiente doméstico inadequado, como piso escorregadio $(26 \%)$, objetos no chão $(22 \%)$ e problemas em degraus $(7 \%)$, entre outras. 0 principal ambiente das quedas foi o próprio lar, em $66 \%$ dos casos, contra apenas $22 \%$ na rua e na casa de parentes ${ }^{18}$.

Convém apontar a existência de seções destinadas ao acompanhamento longitudinal dos valores de pressão arterial sistêmica, controle da glicemia capilar e calendário de imunizações. Por fim, ressalta-se um espaço específico para avaliação de periodicidade anual da saúde bucal e identificação de necessidades de saúde a ela relacionadas pela equipe de saúde bucal presentes no território ${ }^{12}$.

Depreende-se, portanto, que esse instrumento, quando empregado em sua totalidade e de modo adequado, favorece a articulação dos saberes e das práticas na APS, tendo em vista o atendimento integral das necessidades dos usuários. Ponderase, assim, sua concordância com os pressupostos da Política Nacional da Humanização da Atenção e Gestão do SUS (Humaniza-SUS), no que se refere à corresponsabilidade entre os atores presentes no cuidado, ao vínculo entre profissionais e usuários,

\section{...essa ferramenta é instituída enquanto mecanismo para a cidadania e o empoderamento dos sujeitos...}

à garantia de direitos dos usuários enquanto protagonistas no processo de cuidar, uma vez que prevê como imperativo para o bom manejo da saúde da pessoa idosa, seu uso tanto pelas equipes de saúde quanto pelos idosos e por seus familiares e cuidadores $^{9,19}$.

Nessa vertente, outro aspecto positivo relativo à experiência das acadêmicas diante do uso da caderneta diz respeito à possibilidade de socialização das informações educativas obtidas. Tais informações remetem a questões como direitos da pessoa idosa, pontuando o estatuto e as políticas vigentes; às orientações sobre o uso e armazenamento de medicamentos; ao acesso a medicamentos no SUS, explicitando os mecanismos para acesso aos medicamentos e/ou correlatos no âmbito do Programa Farmácia Popular; às etapas pertinentes a uma alimentação saudável; além de orientações sobre saúde bucal, prevenção de quedas, realização de atividades físicas e sexualidade ${ }^{12}$.

De modo semelhante, um estudo nacional revelou, segundo relatos de profissionais da saúde, que, mediante recebimento da caderneta, os idosos aderiram a seu uso com satisfação e sempre que vão ao serviço de saúde eles a levam como se fosse um documento, pois foram orientados pelos enfermeiros, no território, acerca de sua importância e da necessidade de mantê-la sempre à mão ${ }^{20}$.

Pode-se inferir que essa ferramenta é instituída enquanto mecanismo para a cidadania e o empoderamento dos sujeitos, processo por meio do qual as pessoas adquirem domínio sobre suas vidas e obtêm conhecimento para a tomada de decisões acerca de sua saúde e seu bem-estar ${ }^{21,22}$.

Posteriormente, discutindo a aplicabilidade dessa ferramenta com a enfermeira de uma das equipes de saúde, identificou-se a presença de outra potencialidade de sua implementação: o entendimento desse instrumento enquanto ferramenta de gestão na APS. 
Como pressupõe a PNSPI, de fato, a caderneta de saúde da pessoa idosa possibilita a organização do serviço, alinhando-se ao planejamento e à sistematização das ações destinadas à população idosa no território, a partir do acompanhamento dos registros e do (re)conhecimento do perfil e das demandas de cuidado desse grupo por parte das equipes da $\operatorname{ESF}^{23,24}$.

Essas informações são capazes de amparar a estruturação e o preparo de ações de prevenção de agravos e de promoção e reabilitação da saúde na comunidade ${ }^{9}$. Na unidade da ESF em questão, podem ser destacadas as estratégias de educação em saúde sob a forma de grupos educativos, fundamentadas no sentido de elucidar questões que perpassam o viver com limitações e comorbidades que se relacionam ao avanço da idade, para fortalecer o envelhecimento ativo ${ }^{25}$.

Para tanto, as ações do serviço destinadas à população idosa se encontram ancoradas em um projeto de extensão universitária denominado “Envelhecer com Arte e Saúde", vinculado à universidade federal em questão, que vem sendo desenvolvido na unidade da ESF desde o início de 2016, quinzenalmente, com a participação de 12 idosos. Logo, o grupo representa uma proposta interativa e dinâmica, que possibilita a abordagem de diversas temáticas, em um espaço perpassado pela troca de saberes e experiências entre usuários, familiares, cuidadores e profissionais, sob uma perspectiva de autonomização em resposta às dúvidas e necessidades percebidas ${ }^{26}$.

\section{Entraves na implementação do instrumento}

Como explicitado, objetivando aprimorar os cuidados à população idosa, ferramentas como a caderneta de saúde da pessoa idosa foram criadas com base na legislação brasileira. No entanto, percebe-se que importantes desafios ainda se mostram presentes para implementá-la ${ }^{27}$.

Dentre os entraves percebidos pelas acadêmicas nesse processo, pode-se destacar o desconhecimento do significado prático do preenchimento da caderneta, observado na conduta dos profissionais que atuam na APS. Grande parte deles relaciona a caderneta apenas como um instrumento estático para a identificação sociodemográfica desse grupo, sem considerá-la uma ferramenta para o cuidado continuado, distanciando-a, ainda, da prática diária.

Em consonância com a afirmativa acima, um estudo desenvolvido junto aos enfermeiros da APS de

\section{...pode-se destacar o desconhecimento do significado prático do preenchimento da caderneta...}

Caririaçu-CE demonstra que, embora os profissionais estejam cientes de algumas das ações que constam nesse instrumento de monitoramento da saúde do idoso, em nenhum momento o preenchimento da caderneta é citado, de modo específico, como atividade realizada ${ }^{20}$.

Ademais, no que concerne ao preenchimento da caderneta, aponta-se que, apesar de sua estruturação em perspectiva multidisciplinar, esse processo de trabalho compartilhado não foi observado na prática, especialmente pelo entendimento errôneo de muitos profissionais da equipe de saúde de que, em geral, trata-se de uma tarefa exclusiva da enfermagem, deixando de cumpri-la.

Além das atribuições assistenciais, o enfermeiro desempenha uma série de outras atividades, que podem o sobrecarregá-lo enquanto profissional, impedindo-o de dedicar-se exclusivamente àquilo que compete à sua categoria - como é o caso da consulta de enfermagem ${ }^{28}$. No que diz respeito à saúde no país, inúmeros são os profissionais que relatam alta demanda e escassez de tempo para o cumprimento de suas atribuições, o que acaba por comprometer a qualidade do atendimento. Em consequência desses acontecimentos, frequentemente a promoção e prevenção de agravos voltadas à população idosa fica em segundo plano, comprometendo a implementação de ferramentas como a descrita ao longo deste $\operatorname{artigo} 0^{24,29}$.

Outra questão pertinente se relaciona às dificuldades para a realização das visitas no domicílio, ainda que este seja considerado o cenário ideal para o preenchimento da caderneta, como indica $0 \mathrm{MS}^{13}$. São apontados como impeditivos para essa prática a existência de ações programáticas, motivada por excessivo cumprimento de metas, sobrecarga de trabalho, anteriormente citada, e, sobretudo, falta de conhecimentos básicos em geriatria e gerontologia ${ }^{30}$.

Ainda que a população idosa seja frequentadora 
assídua do serviço de saúde, no contexto atual, percebe-se a necessidade de superar uma série de entraves nas unidades daESF, podendo-se citar, ainda, o panorama municipal de constantes substituições nas equipes de saúde, cujo trabalho deveria estar alicerçado na construção e no fortalecimento de vínculos entre o serviço e os idosos, a fim de consolidar o uso da caderneta enquanto ferramenta de cuidado e gestão de saúde.

\section{CONCLUSÃO}

Diante das considerações expostas, destacase importância da caderneta de saúde da pessoa idosa enquanto ferramenta para cuidado e gestão da assistência continuada e rastreio de condições agravantes na população idosa. Nesse sentido, pode-se inferir que cabe ao profissional habilitado multiplicar e fortalecer seu uso na prática, bem como utilizá-la como subsídio para o diagnóstico das necessidades da população e o planejamento de ações de prevenção de agravos e promoção e reabilitação da saúde consideradas pertinentes.

No que diz respeito aos entraves observados no processo de implementação desse instrumento em uma unidade da ESF do município em análise, pontua-se como impeditivos à sua efetivação: a) o desconhecimento dos objetivos e significados de seu uso prático; b) o entendimento da caderneta como mera tarefa a cargo do enfermeiro; c) a sobrecarga de trabalho; e d) as lacunas de conhecimento técnicocientífico nas áreas de geriatria e gerontologia entre os profissionais da equipe de saúde.

A manipulação dessa ferramenta fortaleceu as acadêmicas como profissionais de enfermagem, sob uma perspectiva holística e instrumentalizada que reitera a importância de proporcionar cuidados multiprofissionais e continuados à população. Ademais, destaca-se, em especial, a experiência das visitas domiciliares, que favorecem a aproximação com os usuários, personagens centrais no processo

\section{...caderneta de saúde da pessoa idosa enquanto ferramenta para cuidado e gestão da assistência...}

de cuidar levado a cabo pelos profissionais de enfermagem.

Pode-se apontar como limitações deste estudo o reduzido tempo em campo prático, considerado insuficiente para que os achados sejam passiveis de generalização. Sugere-se que novos estudos sejam conduzidos no campo da enfermagem, de modo a abordar, com maior propriedade, as dificuldades e as potencialidades do uso de ferramentas qualificadoras da assistência - como a caderneta de saúde da pessoa idosa.

\section{CONTRIBUIÇÃO DAS AUTORAS}

Alessandra Schmidt, Maria Eduarda Deitos Vasquez e Caroline Bittencourt contribuíram com a realização da pesquisa, o delineamento do estudo e a redação do manuscrito. Cenir Gonçalves Tier e Vanessa Alvez Mora da Silva contribuíram com a revisão crítica do manuscrito. Bethânia Mesquita Cabeda Maciel contribuiu com a redação e revisão crítica do manuscrito.

\section{REFERÊNCIAS}

1. Faleiros VP. Envelhecimento no Brasil do Século XXI: transições e desafios. Argumentum [serial on the internet]. 2014 [cited 2017 Aug 2];6(1):621. Available from: http://periodicos.ufes.br/ argumentum/article/view/7952

2. Instituto Brasileiro de Geografia e Estatística. Sintese de indicadores sociais (SIS): uma análise das condições de vida da população brasileira 2016. Rio de Janeiro: IBGE; 2016.

3. Küchemann BA. Envelhecimento populacional, cuidado e cidadania: velhos dilemas e novos desafios. Sociedade e Estado [serial on the internet]. 2012 [cited 2017 Aug 1];27(1):165-80. Available from: http://www.scielo.br/pdf/se/v27n1/09.pdf

4. Andrade LM, Sena EL, Pinheiro GM, Meira EC, Lira LS. Políticas públicas para pessoas idosas no Brasil: uma revisão integrativa. Ciênc Saúde Colet [serial on the internet]. 2013 [cited 2017 Aug 3];(18):354352. Available from: https://www.scielosp.org/scielo. php? pid $=$ S1413-81232013002000011\&script $=$ sci abstract \&thng $=p t$

5. Muniz EA, Freitas CASL, Albuquerque IMAN, Linhares MSC. Assistência domiciliar ao idoso no contexto da Estratégia Saúde da Família: análise da produção científica. Sanare (Sobral, 0nline) [serial on the internet]. 2014 [cited 2016 Sep 29];13(2):8691. Available from: https://sanare.emnuvens.com. br/sanare/article/view/578/311 
6. Brasil. Portaria n. 399/GM, de 22 de fevereiro de 2006. Divulga o Pacto pela Saúde 2006 -Consolidação do SUS e aprova as Diretrizes 0peracionais do Referido Pacto [document on the internet]. 2006 [cited 2017 Aug 4]. Available from: http://bvsms.saude.gov.br/ bvs/saudelegis/gm/2006/prt039922 02 2006.html

7. Brasil. Portaria n. 2.048, de 3 de setembro de 2009. Aprova o Regulamento do Sistema Único de Saúde (SUS) [document on the internet]. 2009 [cited 2017 Aug 2]. Available from: http://bvsms.saude.gov. br/bvs/saudelegis/gm/2009/prt2048 $03 \quad 092009$. $\underline{\text { html }}$

8. Brasil. Resolução n. 159/93, de 19 de abril de 1993. Dispõe sobre a consulta de enfermagem [document on the internet]. 1993 [cited 2017 Aug 3]. Available from: http://www.cofen.gov.br/ resoluo-cofen-1591993 4241.html

9. Brasil. Caderneta de Saúde da Pessoa Idosa: manual de preenchimento. Brasília (DF): Ministério da Saúde; 2008.

10. Camarano AA, Kanso S. As instituições de longa permanência para idosos no Brasil. Rev Bras Estud Popul [serial on the internet]. 2010 [cited 2017 Aug 2];27(1):232-5. Available from: http://www.scielo. br/pdf/rbepop/v27n1/14.pdf

11. Tavares DMS, Dias FA. Capacidade funcional, morbidades e qualidade de vida de idosos. Texto \& Contexto Enferm [serial on the internet]. 2012 [cited 2017 Aug 10];1;21(1):233-5. Available from: http://www.scielo.br/pdf/rbepop/v27n1/14.pdf

12. Brasil. Caderneta de Saúde da Pessoa Idosa. Brasília (DF): Ministério da Saúde; 2014.

13. Brasil. Caderneta de Saúde da Pessoa Idosa: manual de utilização. Brasília (DF): Ministério da Saúde; 2016.

14. Bezerra TA, Brito MA, Costa KN. Caracterização do uso de medicamentos entre idosos atendidos em uma unidade básica de Saúde da Família. Cogitare Enferm [serial on the internet]. 2016 [cited 2017 Aug 6];21(1):1-11. Available from: https://revistas. ufpr.br/cogitare/article/view/43011

15. Monteiro ORB, Figueiredo NR, Marreiros MDOC, Figueiredo MDLF, Lima NA, Carvalho Júnior JAM. The occurrence of polypharmacy among elderly assisted by the Family Health Strategy/Polifarmácia entre idosos assistidos pela estratégia saúde da família. Rev Enferm UFPI [serial on the internet]. 2014 [cited 2017 Aug 11];3(2):56-61. Available from: http://www.ojs.ufpi.br/index.php/reufpi/ article/view/1419

16. Lima CAB, Carvalho JL, Aquino RCA. Avaliação de vulnerabilidade do idoso através da adaptação transcultural do instrumento de identificação do idoso vulnerável VES-13. Revista Eletrônica da Estácio Recife [serial on the internet]. 2017 [cited 2017 Aug 9];3(1):[about 7 pages]. Available from: https://reer.emnuvens.com.br/reer/article/view/115

17. Silva IT, PINTO Junior EP, Vilela AB. Autopercepção de saúde de idosos que vivem em estado de corresidência. Rev Bras Geriatr Gerontol [serial on the internet]. 2014 [cited 2017 Aug 9];17(2):27587. Available from: http://www.redalyc.org/ pdf/4038/403838837006.pdf

18. Fabrício SC, Rodrigues RA, Costa Junior ML. Rev Saúde Pública [serial on the internet]. 2004 [cited 2017 Aug 6];(38):93-9. Available from: https://www. scielosp.org/article/rsp/2004.v38n1/93-99/

19. Brasil. Política Nacional de Humanização. Brasília (DF): Ed. Ministério da Saúde; 2004.

20. Barros TB, Maia ER, Pagliuca LMF. Facilidades e dificuldades na assistência ao idoso na Estratégia de Saúde da Família. Rev Rene [serial on the internet]. 2011 [cited 2017 Aug 3];12(4):732-41. Available from: http://www.redalyc.org/html/3240/324027977010/

21. Taddeo PD, Gomes KW, Caprara A, Gomes AM, Oliveira GC, Moreira TM. Acesso, prática educativa e empoderamento de pacientes com doenças crônicas. Ciênc Saúde Colet [serial on the internet]. 2012 [cited 2017 Aug 12];(17):2923-30. Available from: http://www.scielo.br/pdf/csc/v17n11/v17n11a08. pdf

22. Vieira RS, Souza Vieira R. Saúde do idoso e execução da política nacional da pessoa idosa nas ações realizadas na atenção básica à saúde. Rev Direito Sanit [serial on the internet]. 2016 [cited 2017 Aug 11];17(1):14-37. Available from: http:// www.periodicos.usp.br/rdisan/article/view/117042

23. Santos DA, Albuquerque AC. Ações e serviços oferecidos aos idosos atendidos pela atenção primária no município de Campina Grande-PB. Enferm Foco [serial on the internet]. 2013 [cited 2017 Aug 4];4(3-4):146-9. Available from: http:// revista.cofen.gov.br/index.php/enfermagem/ article/view/536

24. Silva KM, Santos SM. A práxis do enfermeiro da Estratégia de Saúde da Família e o cuidado a o idoso. Texto \& Contexto Enferm [serial on the internet]. 2015 [cited 2017 Aug 8];24(1):105-11. Available from: http://www.redalyc.org/pdf/714/71438421013.pdf

25. Machado ARM, Walterlânia SS, Dias FA, Tavares DMS, Munari DB. Potencializando um grupo de terceira idade de uma comunidade rural. Rev Esc Enferm USP [serial on the internet]. 2015 [cited 2017 Aug 15];49(1):96-103. Available from: http://www.scielo.br/scielo.php?script=sci arttext\&pid $=$ S0080-62342015000100096\&lng=en 
26. Neves RT, Laham CF, Aranha VC, Santiago A, Solimar F, Lucia MC. Envelhecimento e doenças cardiovasculares: depressão e qualidade de vida em idosos atendidos em domicílio. Psicol Hosp [serial on the internet]. 2013 [cited 2017 Aug 14];11(2):72-98. Available from: http://pepsic. bvsalud.org/scielo.php?script=sci arttext\&pid $=$ S1677-74092013000200006

27. Fernandes MTO, Soares SM. 0 estado das políticas públicas de atenção ao idoso no Brasil. Rev Esc Enferm USP [serial on the internet]. 2012 [cited 2017 Aug 15];46(6)1493-501. Available from: http:// www.redalyc.org/html/3610/361033321029/

28. Silva KM, Vicente FR, Santos SM. Consulta de enfermagem ao idoso na atenção primária à saúde: revisão integrativa da literatura. Rev Bras Geriatr Gerontol [serial on the internet]. 2014 [cited 2017 Aug 13];17(3):681-7. Available from: http://www. redalyc.org/pdf/4038/403838839020.pdf

29. Coutinho AT, Popim RC, Carregã K, Spiri WC. Integralidade do cuidado com o idoso na Estratégia de Saúde da Família: visão da equipe. Esc Anna Nery Rev Enferm [serial on the internet]. 2013 [cited 2017 Aug 10];17 (4):628-37. Available from: http://www.redalyc.org/pdf/1277/127729351005.pdf

30. Sossai LC, Pinto IC. A visita domiciliária do enfermeiro: fragilidades $x$ potencialidades. Ciênc Cuid Saúde [serial on the internet]. 2010 [cited 2017 Aug 8];9(3):569-76. Available from: http:// ojs.uem.br/ojs/index.php/CiencCuidSaude/article/ view/6856
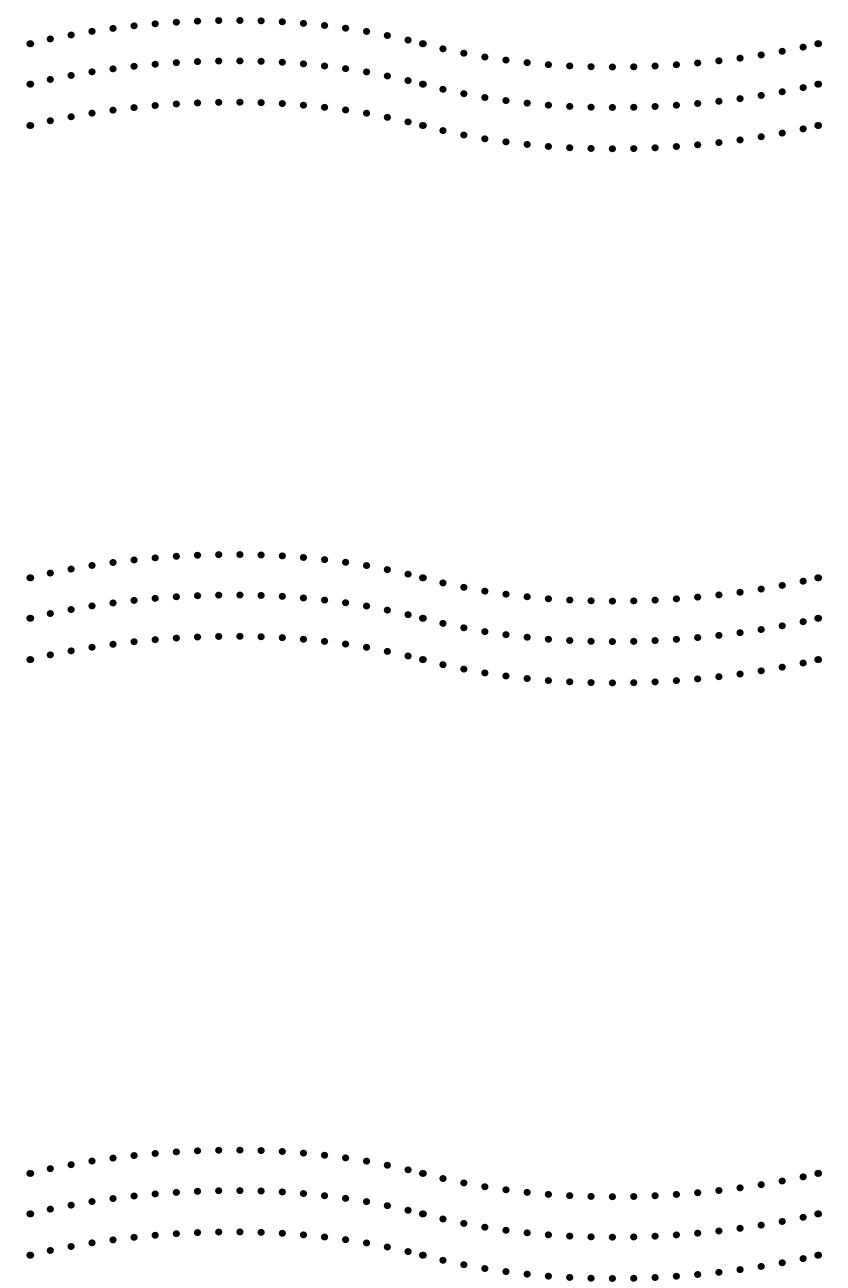
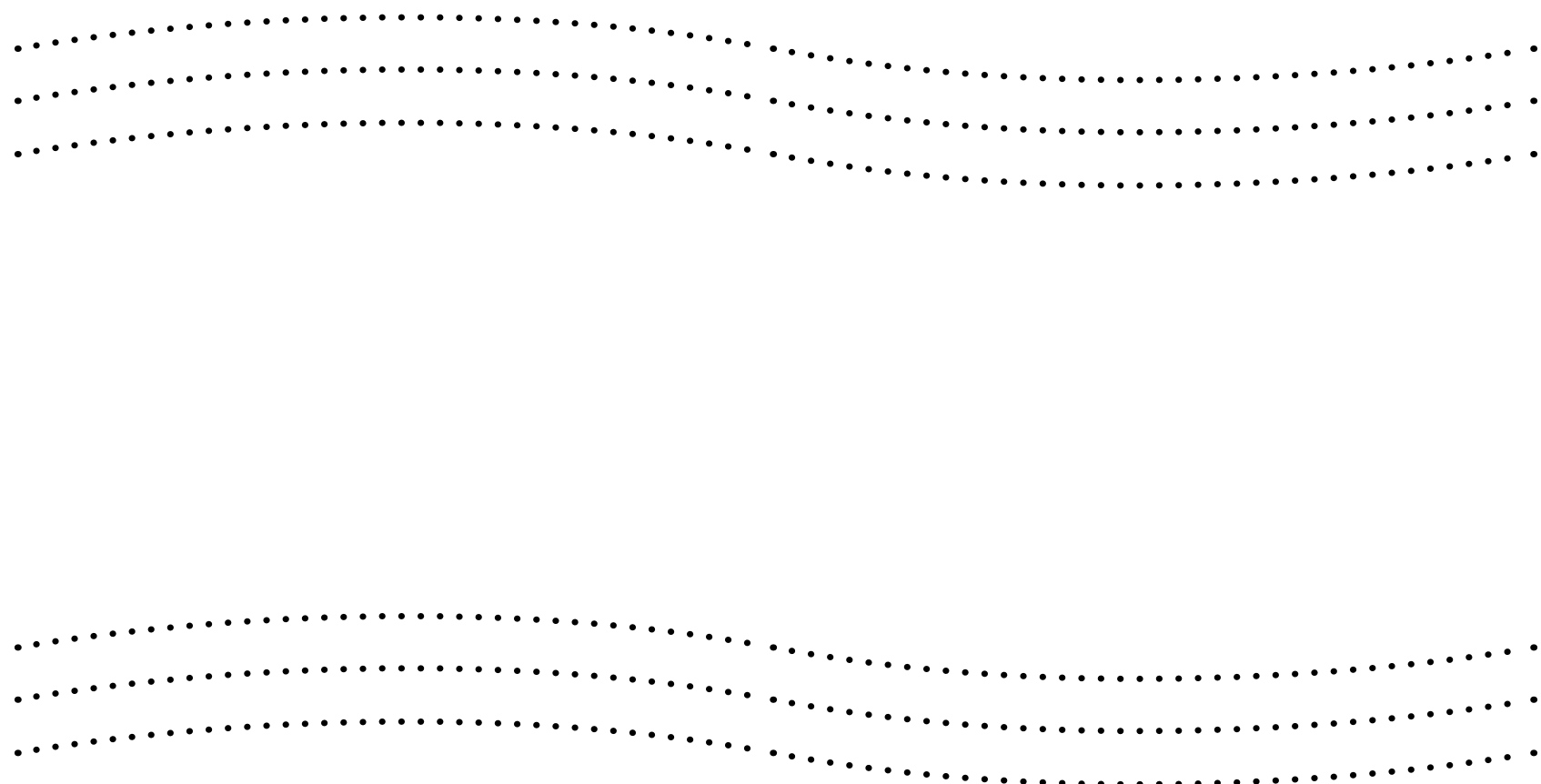